\title{
Contact mechanics of highly porous oxide nanoparticle agglomerates
}

\author{
Andrea Fabre $\cdot$ Samir Salameh • \\ Lucio Colombi Ciacchi • \\ Michiel T. Kreutzer · J. Ruud van Ommen
}

Received: 11 May 2016/Accepted: 4 July 2016/Published online: 18 July 2016

(C) The Author(s) 2016. This article is published with open access at Springerlink.com

\begin{abstract}
Efficient nanopowder processing requires knowledge of the powder's mechanical properties. Due to the large surface area to volume ratio, nanoparticles experience relatively strong attractive interactions, leading to the formation of micron-size porous structures called agglomerates. Significant effort has been directed towards the development of models and experimental procedures to estimate the elasticity of porous objects such as nanoparticle agglomerates; however, none of the existing models has been validated for solid fractions below 0.1 . Here, we measure the elasticity of titania $\left(\mathrm{TiO}_{2}, 22 \mathrm{~nm}\right)$, alumina $\left(\mathrm{Al}_{2} \mathrm{O}_{3}, 8 \mathrm{~nm}\right)$, and silica $\left(\mathrm{SiO}_{2}, 16 \mathrm{~nm}\right)$ nanopowder agglomerates by Atomic Force Microscopy, using a $3.75 \mu \mathrm{m}$ glass colloid for the stress-strain experiments. Three sample preparations with varying degree
\end{abstract}

Electronic supplementary material The online version of this article (doi:10.1007/s11051-016-3500-4) contains supplementary material, which is available to authorized users.

A. Fabre $(\bowtie) \cdot$ S. Salameh $\cdot$ M. T. Kreutzer .

J. R. van Ommen

Department of Chemical Engineering, Delft University of

Technology, Julianalaan 136, 2628 BL Delft,

The Netherlands

e-mail: a.fabre@tudelft.nl

J. R. van Ommen

e-mail: J.R.vanOmmen@tudelft.nl

L. C. Ciacchi

Hybrid Materials Interfaces University of Bremen,

Bremen, Germany of powder manipulation are assessed. The measured Young's moduli are in the same order of magnitude as those predicted by the model of Kendall et al., thus validating it for the estimation of the Young's modulus of structures with porosity above $90 \%$.

Keywords Young's modulus · Porous agglomerates · Atomic force microscopy - Oxide particles · Nanoparticles · Particle characterization · Instrumentation

\section{Introduction}

The mechanical properties of nanopowders are critical for the optimization of their processing (Iwadate and Horio 1998; Bika et al. 2001; Rong et al. 2004). These properties are crucial in gas-phase processes involving agglomerate collision such as in flame aerosol reactors (Kruis et al. 1998; Madler et al. 2006), lung nanoparticle uptake studies (Limbach et al. 2005), and nanopowder fluidization (Matsuda et al. 2004). The processing method of fluidization, where the powder is suspended in a gas stream moving upwards, is known to enhance fluid to solid contact by powder dispersion (Jung and Gidaspow 2002; van Ommen et al. 2012; Dadkhah et al. 2012; Quevedo et al. 2010; Shabanian et al. 2012), which is beneficial for heat and mass transfer, and widely used in gas-solid reaction, granulation, and particle coating, drying, and mixing, among many other applications. Nonetheless, 
nanoparticles (NP) fluidize as clusters called agglomerates (Parveen et al. 2013; Zhou and Li 1999; Khadilkar et al. 2014; Liang et al. 2009), making the dynamics within the fluidized bed complex and challenging to study, mainly due to the lack of accurate characterization of the agglomerates (Salameh et al. 2012).

Nanopowders agglomerate in a stepwise fashion (Yao et al. 2002). During synthesis at high temperatures, primary particles form chemical bonds creating chain-like structures called aggregates, reaching sizes of 100s of nm. These aggregates then cluster together by physical interactions forming simple agglomerates with sizes of a few 10 s of $\mu$ m, mainly during powder storage. Finally, the simple agglomerates assemble into complex agglomerates, which can reach sizes of 100 s of $\mu \mathrm{m}$. As a hierarchical process, each level has structures with particular features such as fractal dimension (de Martin et al. 2014; Hu et al. 2012). This research focuses on the agglomerate properties since these are the structures readily available from stored nanopowder and found during nanopowder processing in the gas phase.

Agglomeration arises from the relatively strong attractive interactions among NPs, which include capillary, solvation, van der Waals, and electrostatic forces (Laube et al. 2015; van Ommen et al. 2012; Castellanos 2005; Hakim et al. 2005; Seville et al. 2000; van Ommen 2012; Quevedo and Pfeffer 2010; Yan et al. 2010). Electrostatic forces diminish in the presence of humidity. In earlier studies, it was shown that physisorbed water molecules situate between the nanoparticles creating an attractive interaction described by a combination of capillary and solvation forces, which can surpass the van der Waals contribution (Salameh et al. 2012; Laube et al. 2015). During nanopowder processing, attractive forces are challenged by external separation forces such as collision (Matsuda et al. 2004). In order to estimate the magnitude of the forces acting on the agglomerates, and thus their morphological stability at the given conditions, their Young's modulus must be known. The high porosity $(>90 \%)$ and rather large size of these structures $(\approx 100 \mu \mathrm{m})$ make them extremely fragile. Therefore, stress measurements to study their mechanical properties are particularly challenging.

Because of their large void fraction, nanoparticle agglomerates are expected to have a relatively low Young's modulus. Due to their fragile nature, any type of manipulation can easily compromise the morphological integrity of the agglomerates and reproducibility of the results. Thus, all techniques considered for the study of agglomerates have to be evaluated for the degree of morphological modification and data reproducibility. Additionally, the number of available techniques to study structures in the micron-size scale with nanoscale resolution is very limited. A quite challenging property to measure is elasticity, mainly due to the structural and technical limitations just mentioned. There have been novel techniques to measure elasticity of soft microscopic objects such as microcapillaries, relying on pressure-induced deformation of microscopic deformable particles in a dilute suspension (Wyss et al. 2010), the ultrasonic pulseecho method by measuring the velocity of ultrasonic waves in materials along a known crystal direction for isotropic, millimeter thick samples (Yoshimura et al. 2007; Schwarz et al. 1997), or the compression and indentation techniques such as atomic force microscopy (AFM). Methods that require the samples to be in the liquid phase, specifically oriented, or placed at a set location will considerably affect the original structure of the nanoparticle cluster and hinder result reproducibility.

Agglomerate elasticity has been measured before; however, those agglomerates had a significantly higher solid fraction, well-defined geometry, and/or customized formation process than those of interest in this research. In 1987, Kendall et al. (1987) measured the elasticity of ceramic NP clusters to study the effect of solid fraction, developing a model to estimate the effective Young's modulus in terms of the volume packing, and particle interface energy, size, and modulus. Nonetheless, the experiments were limited to structures with porosity below $70 \%$ (Kendall et al. 1987). Later on, in 1992, Kendall focused on the elasticity of spray-dried spherical agglomerates of uniformly packed 210-nm zirconia particles (Kendall and Weihs 1992), modeling the steps towards agglomerate fracture and describing the use of a nanoindenter to study agglomerate deformation, again, facing the porosity limitation. In 2001, Bika et al. (2001) presented a summary of studies done on the mechanical properties of wet and dry agglomerates, highlighting their morphological frailty, and the lack of proper measuring techniques and realistic theoretical models to obtain accurate values of the agglomerate mechanical properties. Nonetheless, all the data 
gathered from the literature and reviewed by Bika et al. is for agglomerates with porosity bellow $75 \%$.

The elasticity, represented as the Young's modulus, of porous materials can be predicted from theoretical models found in the literature (Yoshimura et al. 2007). These models consider the agglomerate volume fraction and primary particle Young's modulus as critical variables to determine the agglomerate Young's modulus. However, the models of Hasselman (1962), Wang (1984), Martin and Haynes (1971), and Phani and Niyogi (1987) have fitting parameters that rely on elasticity experimental data, and thus not really predicting the value. The models of Yoshimura et al. (2007) and Yoshimura et al. (2007) require previous knowledge of the shear and bulk modulus of the porous structure, and Poisson's ratio of the NP, which leads to a straightforward calculation of the elasticity. Nonetheless, these values are unknown for nanoparticle agglomerates. Kendall et al. (1987) developed a model with a simple expression that uses the agglomerate solid fraction and NP Young's modulus, work of adhesion, and diameter to estimate the effective elasticity of the porous agglomerate, which can be obtained from commercial suppliers or literature. However, to the best of our knowledge, none of these models has been experimentally validated for structures with porosity above $90 \%$ such as those seen in nanopowders.

A well-established technique to study the elasticity of soft matter is the AFM, which works by forcing an interaction between a probe and the sample. The versatility of the technique allows for the visualization of topographic characteristics to an atomic level, the quantification of interacting forces between nanosized objects, metal deposition on electroconductive substrates, and the measurement of mechanical properties of soft materials (Pimpang et al. 2013; Vakarelski and Higashitani 2006; Barcons et al. 2012; Sigdel et al. 2013; Salameh et al. 2014; Stiles et al. 2008; FarshchiTabrizi et al. 2006; Li and Chen 2014; Salameh et al. 2012; Rong et al. 2004; Webber et al. 2008; Tanabe and Tatsuma 2012). This includes fragile micron/nanosized systems such as muscle cells (Engler et al. 2004) and thin gels (Engler et al. 2004) among many other applications (Picas et al. 2012; Xu et al. 2007; Lin et al. 2007; Zheng and Ya-Pu 2004; Rong et al. 2004; Landolsi et al. 2013; Fotiadis et al. 2002; Rico et al. 2005; Dimitriadis et al. 2002; Salameh et al. 2012). In earlier studies, the AFM equipped with a glass colloid attached to the cantilever was used to measure the
Young's modulus of highly porous NP films (Schopf et al. 2013; Butt et al. 2005). To neglect extra phenomena such as adhesion forces and plasticity, only the approach part of the force curved was fitted to the Hertz model for elasticity estimations. However, these films differ from the fluidized agglomerates on the mechanism of formation, homogeneity, and stability, with porosity still bellow that of the complex nanoparticle agglomerates. This method is widely accepted for materials in the $\mathrm{kPa}-\mathrm{MPa}$ range such as biological samples (Vinckier and Semenza 1998; Roduit C 2009; Radmacher et al. 1996).

The objective of this work is to present an experimental method to measure the elasticity of nanopowder agglomerates, which typically have a porosity above $90 \%$. The results are used to validate the applicability of elasticity models for highly porous structures. Three sample preparation approaches are compared to verify the conservation of the structure, and measurement accuracy and reproducibility. To preserve the original morphology of the agglomerate, the technique requiring the least manipulation during sample preparation is used to investigate hydrophilic titania $\left(\mathrm{TiO}_{2}-\mathrm{P} 25\right)$, alumina $\left(\mathrm{Al}_{2} \mathrm{O}_{3}-\right.$ Alu $\left.\mathrm{C}\right)$, and silica $\left(\mathrm{SiO}_{2}-\mathrm{A} 130\right)$ nanopowders. The experimental results are compared to theoretical models from the literature, and the Kendall et al. (1987) method was found to give a descent estimation.

\section{Experimental section}

\section{Powder characterization}

The nanopowders used in this study are Aeroxide P25 $\left(\mathrm{TiO}_{2}\right)$, Aeroxide Alu C $\left(\mathrm{Al}_{2} \mathrm{O}_{3}\right)$, and Aerosil A130 $\left(\mathrm{SiO}_{2}\right)$, obtained from Evonik with the specifications given in Table 1. To verify the powder characteristics, the primary particle size was determined from TEM images by manually counting 250, 678, and 706 particles for $\mathrm{TiO}_{2}, \mathrm{Al}_{2} \mathrm{O}_{3}$, and $\mathrm{SiO}_{2}$, respectively, using the open source image processing software ImageJ. The mean values obtained are $22 \pm 8,16 \pm 6$, and $8 \pm 2 \mathrm{~nm}$ for $\mathrm{TiO}_{2}, \mathrm{SiO}_{2}$, and $\mathrm{Al}_{2} \mathrm{O}_{3}$, respectively (Fig. 1), where the \pm values are the standard deviation of each dataset. These values agree with those specified by the supplier (Table 1), with the exception of $\mathrm{Al}_{2} \mathrm{O}_{3}$, which showed a significantly lower mean size. The discrepancy could arise from the subjective 
Table 1 Properties of the nanopowders as provided by the manufacturer and obtained from TEM image analysis

\begin{tabular}{lllc}
\hline powder & $\rho_{p}\left(\mathrm{~kg} / \mathrm{m}^{3}\right)$ & $d_{p}(\mathrm{~nm})$ & $d_{p(\mathrm{TEM})}(\mathrm{nm})$ \\
\hline $\mathrm{TiO}_{2}$ P25 & 4000 & 21 & $22 \pm 8$ \\
$\mathrm{Al}_{2} \mathrm{O}_{3}$ AluC & 3800 & 13 & $16 \pm 6$ \\
$\mathrm{SiO}_{2}$ A130 & 2200 & 16 & $8 \pm 2$ \\
\hline
\end{tabular}

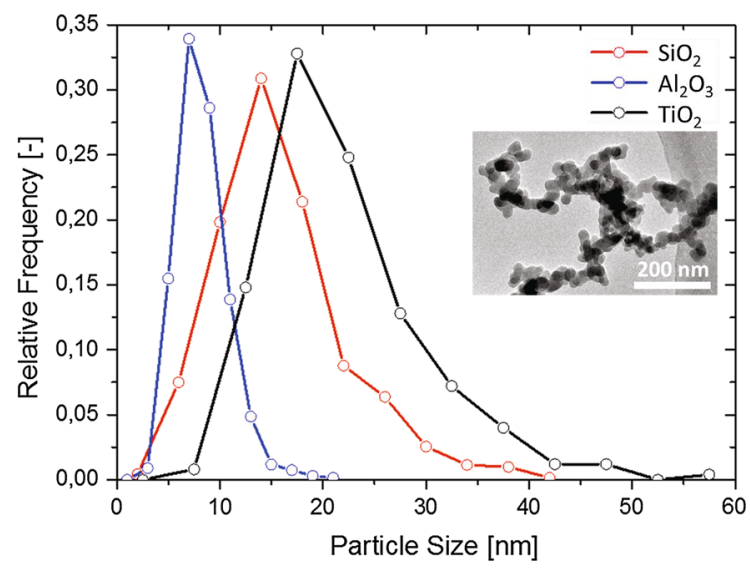

Fig. 1 Size distribution of nanoparticles measured from TEM images using ImageJ. 250, 678, and 706 particles were counted for $\mathrm{TiO}_{2}, \mathrm{Al}_{2} \mathrm{O}_{3}$, and $\mathrm{SiO}_{2}$, respectively. The inset is a TEM image of $\mathrm{Al}_{2} \mathrm{O}_{3}$ showing nanoparticles connected by solid necks

particle selection during image analysis by measuring only those shades that clearly seem to be individual particles, as most of them are connected by solid necks (Fig. 1 inset). Also, the inconsistency could come from the use of different measuring techniques since the average size given from production is determined by the gas adsorption-desorption method, which could deviate from that obtained from the TEM image analysis.

\section{Sample preparation}

Three sample preparation methods were tested, referred to as pressed on glass, double-sided tape, and rough substrate. For the powder pressed on glass, a small amount of the nanopowder is placed on a glass microscope slide over an area of about $1 \mathrm{~cm}^{2}$, and pressed using a thick piece of flat glass until leaving a homogeneous layer of powder looking uniform to the naked eye. The force exerted over the film is estimated using a scale reaching $12 \pm 1 \mathrm{~N}$, which results in a pressure of $9 \pm 0.5 \mathrm{kPa}$. The double-sided tape method involves the spreading of powder over a transparent double-sided tape (Scotch) attached to a glass slide. Then, the sample is gently shaken to remove any loose powder without blowing or touching, to prevent morphological changes. Similarly, the rough surface preparation starts with the spreading of powder on the rough side of a microscope slide, with a final gentle shake to remove the excess powder. These spreading and shaking steps are repeated a few times to ensure a thick enough powder layer for AFM measurements. Due to the extent of manipulation, the pressed on glass method deliberately modifies the structure of the powder, while the sample on the rough substrate is expected to have an almost unchanged morphology. Comparably, the double-sided tape technique is believed to preserve the original structure of the agglomerates. However, the effect of the glue on the mechanical properties was questionable, and thus evaluated.

All three samples were characterized by SEM imaging. A SEM (Jeol JSM-6010 LA) was used to evaluate the general morphology of the nanopowder film on the smooth glass, rough surface, and doublesided tape. To assess the glue-powder integration, images of the tilted double-sided tape sample were taken and analyzed. The samples were slightly blown to prevent nanopowder contamination of the sample chamber. Additionally, for clear SEM imaging, the samples were coated with gold using the Auto Sputter Coater (JEOL JFC-1300).

\section{Elasticity measurements}

The stress-strain measurements were done in a Nanowizard 3 AFM from JPK. The experiments were performed using a probe with a glass colloid of $3.5 \mu \mathrm{m}$ in diameter bought from sQube (CP-FM-SiO-B) (see Fig. S1, Supporting Information). This colloid size is large enough to prevent local indentation through the primary particles, and apply pressure on an area encompassing nanoparticles agglomerates. The spring constants of 2.6, 3.5, and $3.9 \mathrm{~N} / \mathrm{m}$ for $\mathrm{Al}_{2} \mathrm{O}_{3}, \mathrm{SiO}_{2}$, and $\mathrm{TiO}_{2}$ on double-sided tape, respectively, and 3.8 and $4.4 \mathrm{~N} / \mathrm{m}$ for $\mathrm{TiO}_{2}$ on a rough substrate and pressed on glass, respectively, were determined using the thermal noise method (Hutter and Bechhoefer 1993; Burnham et al. 2003). Single force curves were recorded on $8 \times$ 8 grids in an area of $10 \times 10 \mu \mathrm{m}$ to average local differences. The applied force was $150 n N$ with a cantilever approach/retraction speed of $2 \mu \mathrm{m} / \mathrm{s}$. To 
avoid glue-colloid contact, the agglomerates were located before each stress-strain experiment by a microscope positioned right below the sample (Fig. 2).

The Young's modulus was calculated using the JPKSPM Data Processing software by fitting the Hertz model (Hertz 1881) to the approach curves. First, the baseline was subtracted from the curves to set the point of cantilever-sample contact at zero and have displacement equal to indentation. Then, the $\mathrm{x}$ offset (contact point) was adjusted and the height for cantilever bending, corrected previous to the Young's modulus estimation using the embedded "determine elasticity from indentation" software function. Retraction curves were not considered for elasticity measurements of the agglomerates; hence, only the approach curves are presented and used for the estimation of the Young's modulus. Other contact mechanics models such as Johnson-Kendall-Roberts (JKP) (Johnson et al. 1971), Derjaguin-Muller-Toporov (DMT) (Derjaguin et al. 1975), and MaugisDugdale (MD) (Maugis 1992), which account for adhesion forces (Lin et al. 2007; Landolsi et al. 2013), were also considered.

\section{Results and discussion}

Sample characterization

From the TEM pictures, it is evident that nanoparticles are found in clusters. These structures are very porous and expected to be susceptible to changes by external disturbances. Therefore, any powder manipulation and processing will dramatically modify their original morphology. Insufficient analysis and understanding of the handling effect can lead to erroneous conclusions regarding the nature of the nanoparticle clusters.

Sample preparation was thoroughly evaluated to prevent false conclusions due to the fragility of the agglomerates. The soft spreading and gentle shake for the rough surface and double-sided tape sample preparation methods show fluffy structures, as expected from unprocessed nanopowder (Fig. 3a, b). On the other hand, the powder pressed on glass shows a flat and dense surface arising from the pressing step (Fig. 3c). Nonetheless, the pressed film seems to keep a highly porous morphology underneath the flat surface (Fig. 3d). The SEM images showed a morphology similar to naturally formed complex agglomerates for the rough surface and double-sided tape samples, while there was considerable modification on the pressed on glass nanopowder film.

Besides the preparation method, the sample substrate could also affect the AFM measurements. From SEM images, it was seen that the powder film thickness is considerably larger than the colloid indentation depth (Fig. 3), and since the elasticity of the solid substrates is known to be orders of magnitude higher than that of the porous film, the substrates should not have an effect on the measurements. However, the possibility of glue penetration by capillary into the highly porous structures led to extra evaluation of samples placed on the double-sided tape. These samples were assessed by tilted SEM imaging, where the glue was found to immerse less than $0.5 \mu \mathrm{m}$ of the attached agglomerates (Fig. 3b). Thus, the glue, as well as the solid substrates, is expected to have negligible to no influence on the AFM measurements,
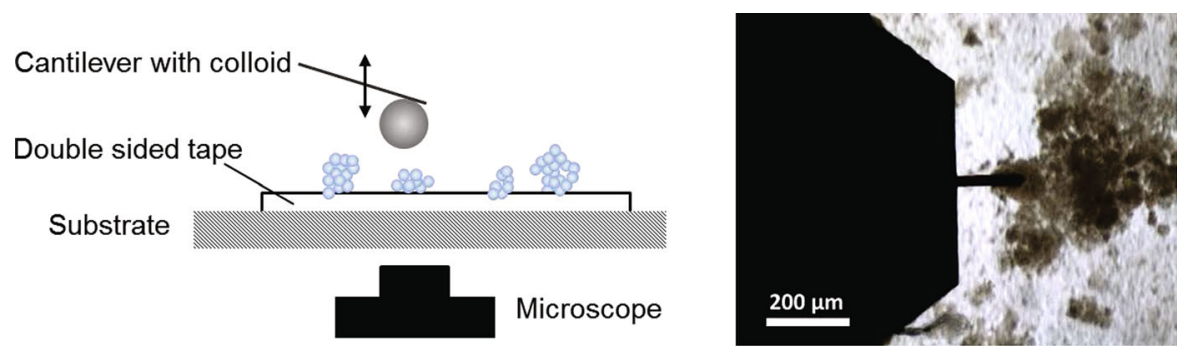

Fig. 2 Schematic representation of the experimental setup for the double-sided tape sample preparation method. The nanopowder is attached to a glass microscope slide (substrate) using transparent double-sided tape. The $3.5-\mu \mathrm{m}$ colloid attached to the cantilever is aligned to the desired position on the sample with the help of a microscope located directly below the sample before each elasticity measurement. The image on the right is of $\mathrm{Al}_{2} \mathrm{O}_{3}$ on double-sided tape, taken by the AFM microscope 

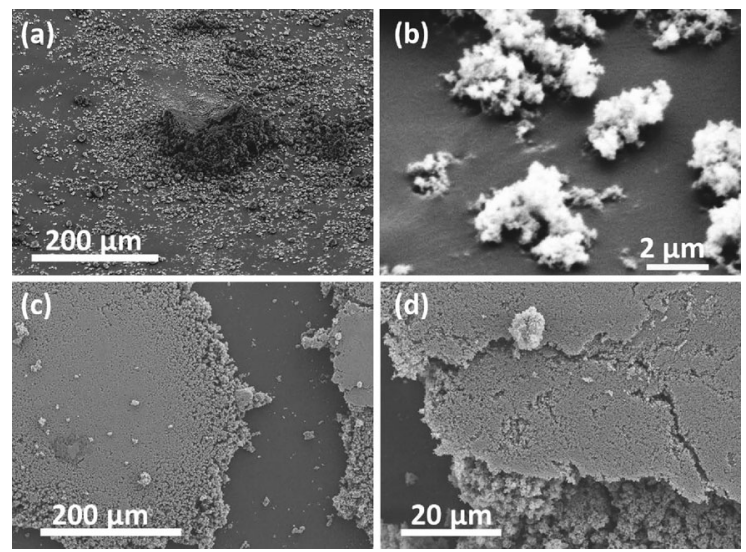

Fig. 3 SEM pictures of $\mathrm{TiO}_{2}$ nanopowder samples; $\mathbf{a}$, b spread on double-sided tape; c, d pressed on glass. The porosity, distribution, and morphology of the powder clusters are noticeably different between the double-sided tape and pressed on glass samples. Images (a, b) show the agglomerates attached to the double-sided tape. The pressed powder cluster shows a very uniform flat surface with a few loose agglomerates on top (c), and an edge that resembles the structure of the spread powder (d)

leaving any measurement discrepancy to the preparation method itself.

Force curve analysis

For an ideal elastic sample, the slope of approach and retraction part does not differ. However, in the case of the highly porous agglomerates, there is a large hysteresis between approach and retraction (Fig. 4a). To investigate the elasticity of porous samples by AFM, the approach part of the force curve should be analyzed (Butt et al. 2005). This is due to the complexity of the retraction curve, which includes other phenomena such as strong short-range adhesion forces between the colloid and the agglomerate that lead to deformation of the agglomerate while the cantilever retracts. Moreover, a certain amount of approach curves $(<33 \%)$ show plastic deformations and an inaccurate fit of the Hertz model (Fig. 4b), and hence were eliminated from data analysis (see Figs. S2 and S3, Supporting Information).

The approach part of the force curves obtained from the AFM measurements were fitted using the Hertz model (Hertz 1881) to calculate the Young's modulus of each sample (Fig. 5a). A total of five samples were analyzed, consisting of $\mathrm{Al}_{2} \mathrm{O}_{3}, \mathrm{SiO}_{2}$, and $\mathrm{TiO}_{2}$ on
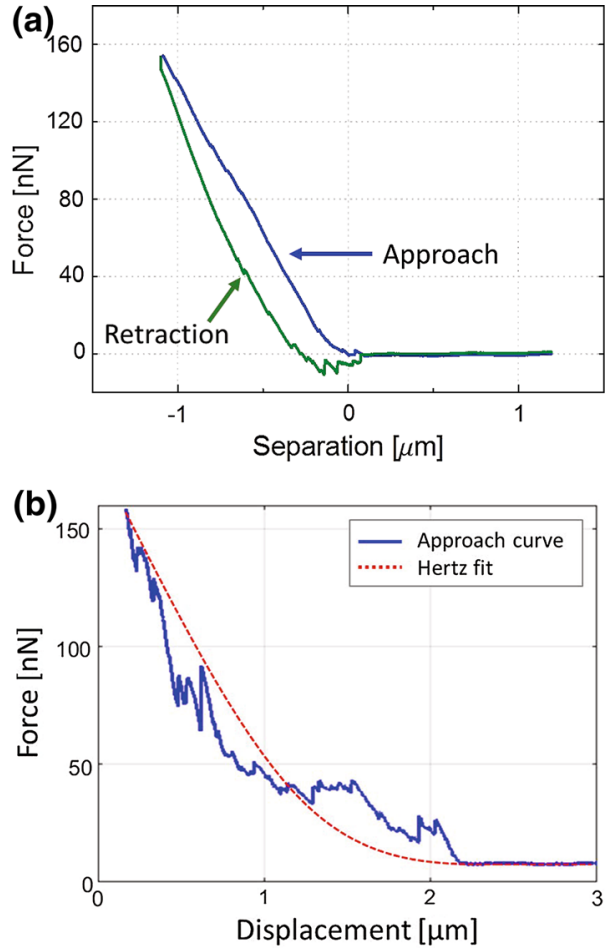

Fig. 4 a Full force curve of an alumina $\left(\mathrm{Al}_{2} \mathrm{O}_{3}\right)$ sample measured by AFM. The approach part of the curve is shown in blue and the retraction part, in green. The horizontal axis is the separation between the colloid and the sample. The hysteresis between the approach and retraction curves, in addition to the multiple peaks on the retraction curve, suggests elastic deformation of the sample. b Force versus displacement curve showing particle rearrangement. Example of a plot not included in the analysis. (Color figure online)

double-sided tape, $\mathrm{TiO}_{2}$ on a rough surface, and $\mathrm{TiO}_{2}$ pressed on glass. None of the samples showed measurable long distance adhesion forces towards the colloid; therefore, models such as DMT, JKR, and $\mathrm{MD}$, which require adhesion for proper fitting, were excluded (Fig. 5c).

The Hertz model is described by the following equation:

$F_{\text {Hertz }}=\frac{4}{3} \frac{E^{*}}{1-v^{* 2}} R_{T i p}^{\frac{1}{2}}\left(s_{0}-s\right)^{\frac{3}{2}}$,

where $E^{*}$ and $v^{*}$ are the Young's modulus and Poisson's ratio of the powder sample, respectively; $R_{\text {Tip }}$ is the radius of the glass colloid, $s_{0}$ is the colloidsample contact distance, and $s$ is the penetration depth. The Hertz model assumes an isotropic and linear elastic solid sample occupying an infinitely extending 

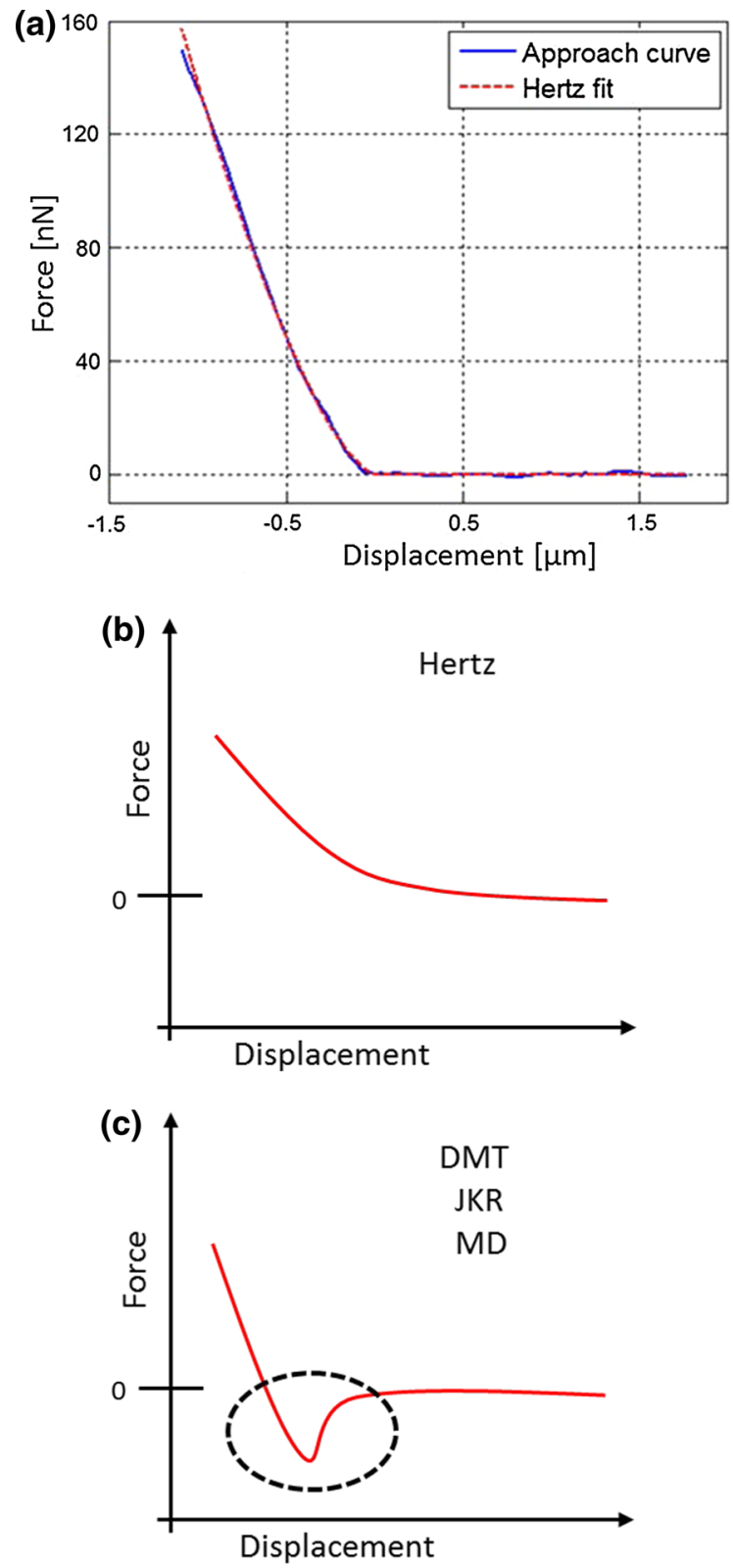

Fig. 5 Hertz fit to the approach part of an experimental force versus displacement curve (a). Hertz contact model curve in a force versus displacement plot (b). General force versus displacement curve for the DMT, JKR, and MD models, which account for the effect of adhesion (c)

half space, nondeformable indenter, no additional interactions between the indenter and sample, negligible indentation compared to the sample thickness, absolute elastic behavior, and a homogeneous sample (JPK 2009). Relative to the soft powder films, the indenter is considered nondeformable. Furthermore, the approach curves used for elasticity analysis did not show additional interactions between the colloid and the film.

The calculated Young's modulus for the three sample preparation methods agrees with the hypothesis developed based on the level of powder manipulation (Fig. 6). For the three $\mathrm{TiO}_{2}$ samples, 115, 155, and 258 curves were measured for the pressed on glass, rough substrate, and double-sided tape, respectively. Due to the inhomogeneity of the film, hundreds of measurements were taken to obtain a statistical representation of each sample. The lower values correspond most likely to film spots far from the ideal Hertz assumptions where the measured location had a lower concentration of agglomerates with a nonuniform solid distribution, which explains the wide distribution, while the higher ones are probably closer to the elastic Young's modulus. The moduli of the double-sided tape and rough substrate are in the same order of magnitude, with a slightly wider distribution for the rough substrate, and the maximum and minimum values similar to those of the double-sided tape. Nonetheless, the pressed on glass sample has a Young's modulus more than one order of magnitude higher (Fig. 6) as a consequence of the denser film made by pressing. The pressed on glass sample also

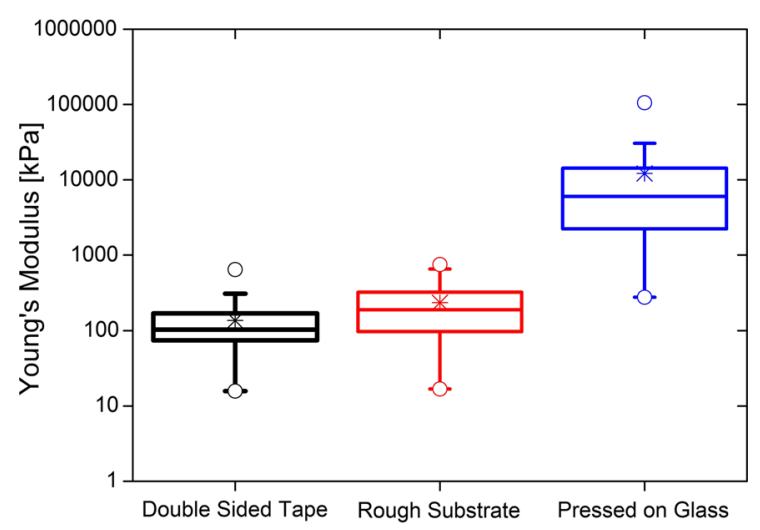

Fig. 6 Young's modulus of $\mathrm{TiO}_{2}$ measured using different sample preparation methods. The double-sided tape and rough substrate preparation methods consist of powder spreading on substrate, and gentle shaking to remove excess powder. The pressed on glass method squeezes the powder between two flat pieces of glass. The asterisks are the mean values and the empty circles are the maximum and minimum values. The box encompasses the second and third quartiles, divided by a line corresponding to the median. The top and bottom whiskers are the outliers with coefficient 1.5 
shows a wider distribution, which could arise from the loose agglomerates present on the surface (Fig. 3b), or any film defect caused by uneven compression or irregular release behavior. Therefore, we have selected the double-sided tape technique as the most reliable sample preparation method.

\section{Elasticity of different materials}

The elasticity of the nanopowder depends on the particle packing, size distribution, shape, surrounding conditions, powder processing, and contact forces. Three common oxide nanopowders of different materials and primary particle sizes were studied, namely $\mathrm{Al}_{2} \mathrm{O}_{3}, \mathrm{SiO}_{2}$, and $\mathrm{TiO}_{2}$. The packing density depends on the size distribution, powder processing, and particle shape, affecting the space available for particle rearrangement. Additionally, the interparticle forces are affected by material properties such as the Hamaker coefficient and surface hydrophobicity. Thus, each of the three powders has an elasticity which depends on the unique material properties and particle morphology.

The Young's modulus of the powders was calculated by fitting the Hertz model to 219, 305, and 158 curves for $\mathrm{SiO}_{2}, \mathrm{Al}_{2} \mathrm{O}_{3}$, and $\mathrm{TiO}_{2}$, respectively. Two of the materials, $\mathrm{Al}_{2} \mathrm{O}_{3}$ and $\mathrm{TiO}_{2}$, showed a Young's modulus within the same range in the order of $100 \mathrm{kPa}$, while $\mathrm{SiO}_{2}$ was an order of magnitude lower (Fig. 7), and with a noticeably narrower distribution. The low Young's modulus means that the $\mathrm{SiO}_{2}$ agglomerate layer is easier to compress. During the measurements, the force applied on the sample by the colloid is specified; this force is directly proportional to the Hamaker coefficient and elastic deformation of the sample (Tsai et al. 1991). Since the Hamaker coefficient of silica $\left(\mathrm{SiO}_{2}\right)$ is about an order of magnitude smaller than that of $\mathrm{Al}_{2} \mathrm{O}_{3}$ and $\mathrm{TiO}_{2}$ (Bergstrom 1997), a more prominent deformation was expected and indeed obtained, describing a soft, highly elastic material. Furthermore, other factors such as the degree of particle surface roughness and porosity could contribute to the low Young's modulus of $\mathrm{SiO}_{2}$, and should be further investigated.

As seen from Fig. 7, the values show a wide distribution. This distribution is typical for AFM measurements. Even in the case of perfectly flat substrates such as mica or silicon, AFM values always show wide distribution based on a different number of

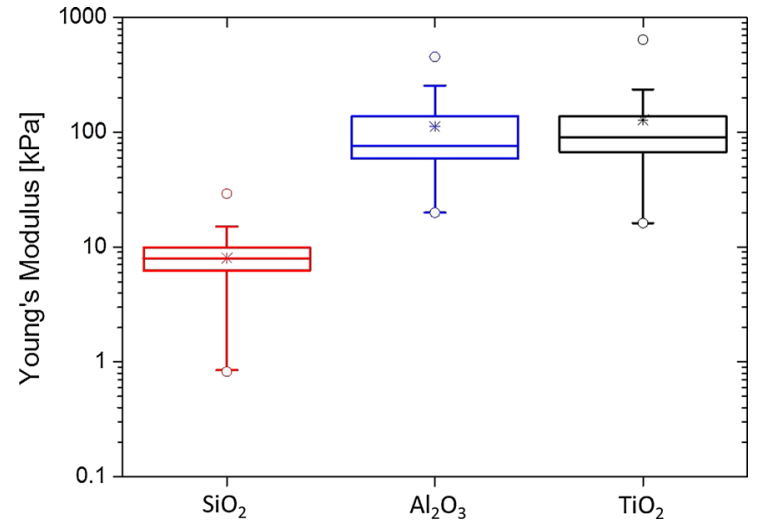

Fig. 7 Young's modulus measured for $\mathrm{TiO}_{2}, \mathrm{SiO}_{2}, \mathrm{Al}_{2} \mathrm{O}_{3}$ on double-sided tape

molecules coming into contact at each measurement (Butt et al. 2005; Farshchi-Tabrizi et al. 2006). However, in the case of porous agglomerates, the contact scenario shows even more variation between measurements since the sample surface is rather heterogeneous, leading to a wider range of measured values.

Theoretical elasticity

The elasticity of each powder was calculated theoretically using the model of Kendall et al. (1987). According to Kendall's model, the effective elasticity of the powder sample can be estimated from

$E^{*}=17.1 \phi^{4}\left[\frac{E^{2} \Gamma}{d_{p}}\right]^{1 / 3}$,

where $\phi$ is the solid volume fraction, and $E, \Gamma$, and $d_{p}$, the Young's modulus, work of adhesion, and diameter of the nanoparticles, respectively. This model was developed for anisotropic packing of spherical particles into complex structures with different shapes. All shapes fell into one curve represented by Eq. (2) where the coefficient 17.1 is found from the fit. The dependence of the effective Young's modulus on the solid fraction to the fourth power arises from a square dependence on the shear modulus $(G)$, and a second one on the coordination number $\left(N_{C o}\right)$.

The applicability of the model to highly porous nanoparticle agglomerates was evaluated. The coordination number of porous structures with solid fraction between 0 and 0.1 still has a square dependence as estimated from the literature (Norman 1971). The original expression to calculate the coordination 
number is an exponential sum that leads to the Taylor series $\quad N_{C o}=1.99+0.59 \phi+11.02 \phi^{2}-0.02 \phi^{3}+$ $10.27 \phi^{4}+O\left(\phi^{5}\right)$, which results in a parabola for small $\phi$. Additionally, the square influence of the density packing on the modulus described for the material with a random distribution of isolated spherical holes (Mackenzie 1950) could still apply to highly porous structures with randomly distributed particle chains such as nanoparticle agglomerates. A Taylor expansion of the original formula reads as

$G=\sum_{n=1}^{\infty} \frac{4 \times 3^{n-1} k_{0}^{n} \mu_{0} \phi^{n}}{\left(3 k_{0}+4 \mu_{0}\right)^{n}}$,

where $k_{0}$ is the bulk modulus and $\mu_{0}$ the shear modulus, which can be taken as a quadratic polynomial for solid fractions in the nanoparticle agglomerate range since terms with higher degrees lead to values more than two orders of magnitude smaller. Therefore, we believe that Kendall's model can be used to estimate the elasticity of structures with solid fractions lower than 0.1 such as the highly porous nanopowder layers presented in this work.

For hydrophilic $\mathrm{TiO}_{2}(\mathrm{P} 25)$, with a solid fraction of 0.03 (Tahmasebpoor et al. 2013), work of adhesion of $0.8 \mathrm{~J} / \mathrm{m}^{2}$ (Navrotsky 2003; Kendall et al. 1987), particle diameter of $22 \mathrm{~nm}$ (Fig. 1), and particle elasticity of $234 \mathrm{GPa}$ (Chen et al. 2009), we obtain a Young's modulus of $174 \mathrm{kPa}$, which is in close agreement with the results from the AFM. The values used for $\mathrm{SiO}_{2}$ (A130) and $\mathrm{Al}_{2} \mathrm{O}_{3}$ (AluC) are shown in Table 2, resulting in Young's moduli of 10 and 129 $\mathrm{kPa}$, respectively. The work of adhesion is calculated as twice the surface energy of the material, which is taken from Navrotsky's paper (Navrotsky 2003). Since the estimation of surface energy depends on the experimental method and conditions showing strong variations in literature, the paper of Navrotsky et al. was chosen as it includes all three powders used in this study. The theoretical and experimental values are compared in Fig. 8, where the empty circles correspond to the theoretical values with bars representing the spread arising from the nanoparticle size distribution, and the solid circles representing the experimental mode with error bars as the standard deviation for log-normal distribution of the data.

Kendall's model can estimate the elasticity of the highly porous sample to the right order of magnitude, which is known to be extremely challenging. The
Table 2 Values used for the estimation of the effective Young's modulus

\begin{tabular}{llllr}
\hline material & $\phi^{\mathrm{a}}$ & $\Gamma\left(\mathrm{J} / \mathrm{m}^{2}\right]^{\mathrm{b}}$ & $E(\mathrm{GPa})^{\mathrm{c}}$ & $d_{p}(\mathrm{~nm})$ \\
\hline $\mathrm{TiO}_{2}-\mathrm{P} 25$ & 0.03 & 0.8 & 234 & $22 \pm 8$ \\
$\mathrm{Al}_{2} \mathrm{O}_{3}-\mathrm{AluC}$ & 0.02 & 5.2 & 400 & $8 \pm 2$ \\
$\mathrm{SiO}_{2}-\mathrm{A} 130$ & 0.02 & 0.18 & 70 & $16 \pm 6$ \\
\hline
\end{tabular}

${ }^{a}$ Solid volume fraction (Tahmasebpoor et al. 2013)

b Nanoparticle work of adhesion (Navrotsky 2003)

c Nanoparticle Young's modulus (Chen et al. 2009; Kendall et al. 1987)

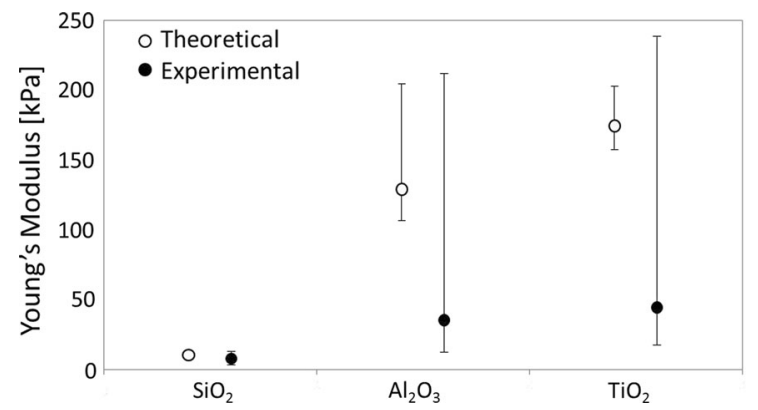

Fig. 8 Experimental and theoretical values of the Young's moduli for $\mathrm{SiO}_{2}, \mathrm{Al}_{2} \mathrm{O}_{3}$, and $\mathrm{TiO}_{2}$ on double-sided tape. Experimental values obtained from AFM measurement (solid circles), and theoretical from Eq. (2) (empty circles) are shown. Error bars are the standard deviation of the curves used to calculate the log-normal experimental elasticity, and the standard deviation from the nanoparticle size distribution as estimated from TEM images

slight discrepancy between the theoretical and experimental Young's modulus values can be attributed to the partial plasticity of the agglomerates and the rough distribution of powder throughout the film. In all three cases, the experimental value is lower than the theoretical one since plastic deformation based on aggregate rearrangements during agglomerate compression by the colloid is not accounted for in Kendall's model. This plasticity of the system must be too small (compared to the spring constant of the cantilever) for the experimental equipment and data analysis software to find the elastic Herzt model unsuited. Furthermore, the spread of the measured values also represents the range of agglomerate properties found throughout the film. 
The parameters needed to calculate the elasticity for the different sample preparation methods are presumably known for the rough substrate and double-sided tape, and assumed to be the same; however, the porosity of the pressed on glass sample is unknown. A porosity of $91 \pm 5 \%$ was back-calculated from the Kendall model for $\mathrm{TiO}_{2}$ pressed on glass sample using the AFM measurements as the effective Young's modulus (Fig. 6). This means that even after the squeezing step, the pressed powder shows a highly porous morphology, which from the SEM images (Fig. 3c) seems appropriately described by the estimated value.

Other theoretical models to compute the Young's modulus were considered (Adachi et al. 2006; Wagh et al. 1991; Pabst et al. 2006; Kupkova 1993; Yoshimura et al. 2007; Choren et al. 2013). Nevertheless, some of them include fitting parameters that require experimental data (Hasselman 1962; Wang 1984; Martin and Haynes 1971; Phani and Niyogi 1987; Choren et al. 2013), which defeats the purpose of the analytical calculation for this study, and those from Yoshimura et al. (2007) use as parameters properties of the porous material that are still unknown due to technical limitations similar to those encountered for the Young's modulus (Kovacik 2001). Alternative models, listed in Choren et al.'s review (Choren et al. 2013), which only depend on agglomerate porosity and Young's modulus of the nonporous material estimate moduli in the gigapascal (GPa) range (see Fig. S4, Supporting Information), more than four orders of magnitude higher than the experimental values obtained from the AFM.

\section{Conclusions}

The research presented in this paper describes a method to experimentally determine the Young's modulus of structures with porosity higher than 90 $\%$. The focus of the study is on nanoparticle agglomerates, which are a few hundred micrometers in size and very fragile, formed due to strong attractive interactions among the primary particles. The experiments are done by AFM on five different samples including three materials $\left(\mathrm{Al}_{2} \mathrm{O}_{3}, \mathrm{SiO}_{2}, \mathrm{TiO}_{2}\right)$ using the double-sided tape sample preparation method, and three sample preparation methods (pressed on glass, rough surface, double-sided tape) for one of the nanopowders $\left(\mathrm{TiO}_{2}\right)$. The results validate the applicability of Kendall et al. model to predict the elasticity of nonspherical highly porous structures. A more detailed analysis on the extrapolation of Kendall's model to low solid fractions and/or irregularly shaped particles will lead to a better understanding of the solid fraction's effect on the effective elasticity of porous structures. The proposed experimental technique can be used for validation of current or future models.

Acknowledgments The research leading to this paper has received funding from the European Research Council under the European Union's Seventh Framework Programme (FP/20072013)/ERC Grant, agreement No. 279632.

Open Access This article is distributed under the terms of the Creative Commons Attribution 4.0 International License (http:// creativecommons.org/licenses/by/4.0/), which permits unrestricted use, distribution, and reproduction in any medium, provided you give appropriate credit to the original author(s) and the source, provide a link to the Creative Commons license, and indicate if changes were made.

\section{References}

Adachi J, Kurosaki K, Uno M, Yamanaka S (2006) Porosity influence on the mechanical properties of polycrystalline zirconium nitride ceramics. J Nucl Mater 358:106-110

Barcons V, Verdaguer A, Font J, Chiesa M, Santos S (2012) Nanoscale capillary interactions in dynamic atomic force microscopy. J Phys Chem C 116(14):7757-7766. doi:10. 1021/jp2107395

Bergstrom L (1997) Hamaker constants of inorganic materials. Adv Colloid Interface Sci 70:125-169. doi:10.1016/ S0001-8686(97)00003-1, URL:http://www.sciencedirect. com/science/article/pii/S0001868697000031

Bika DG, Gentzler M, Michaels JN (2001) Mechanical properties of agglomerates. Powder Technol 117(12):98-112. doi:10. 1016/S0032-5910(01)00318-7, URL:http://www.sciencedir ect.com/science/article/pii/S0032591001003187

Burnham NA, Chen X, Hodges CS, Matei GA, Thoreson EJ, Roberts CJ, Davies MC, Tendler SJB (2003) Comparison of calibration methods for atomic-force microscopy cantilevers. Nanotechnology 14(1):1-6

Butt HJ, Cappella B, Kappl M (2005) Force measurements with the atomic force microscope: technique, interpretation and applications. Surf Sci Rep 59:1-152

Castellanos A (2005) The relationship between attractive interparticle forces and bulk behaviour in dry and uncharged fine powders. Adv Phys 54(4):263-276

Chen B, Zhang H, Dunphy-Guzman KA, Spagnoli D, Kruger MB, Muthu DVS, Kunz M, Fakra S, Hu JZ, Guo QZ, Banfield JF (2009) Size-dependent elasticity of nanocrystalline titania. Phys Rev B 79:125-406. doi:10.1103/ PhysRevB.79.125406 
Choren J, Heinrich S, Silver-Thorn M (2013) Young's modulus and volume porosity relationships for additive manufacturing applications. J Mater Sci 48(15):5103-5112. doi:10. 1007/s10853-013-7237-5

Dadkhah M, Peglow M, Tsotsas E (2012) Characterization of the internal morphology of agglomerates produced in a spray fluidized bed by $\mathrm{x}$-ray tomography. Powder Technol 228:349-358

de Martin L, Bouwman W, van Ommen JR (2014) Multidimensional nature of fluidized nanoparticle agglomerates. Langmuir 30(42):12696-12702. doi:10.1021/la502987e

Derjaguin B, Muller V, Toporov Y (1975) Effect of contact deformations on the adhesion of particles. J Colloid Interface Sci 53(2):314-326. doi:10.1016/0021-9797 (75)90018-1, URL:http://www.sciencedirect.com/science/ article/pii/0021979775900181

Dimitriadis EK, Horkay F, Maresca J, Kachar B, Chadwick RS (2002) Determination of elastic moduli of thin layers of soft material using the atomic force microscope. Biophys $\mathbf{J}$ 82:2798-2810

Engler A, Richert L, Wong J, Picart C, Discher D (2004) Surface probe measurements of the elasticity of sectioned tissue; thin gels and polyelectrolyte multilayer films:correlations between substrate stiffness and cell adhesion. Surf Sci 570:142-154

Farshchi-Tabrizi M, Kappl M, Cheng YJ, Gutmann J, Butt HJ (2006) On the adhesion between fine particles and nanocontacts: an atomic force microscope study. Langmuir 22(5):2171-2184

Fotiadis D, Scheuring S, Muller SA, Engel A, Muller DJ (2002) Imaging and manipulation of biological structures with the AFM. Micron 33(4):385-397. doi:10.1016/S0968-4328 (01)00026-9, URL:http://www.sciencedirect.com/science/ article/pii/S0968432801000269

Hakim LF, Portman JL, Casper MD, Weimer AW (2005) Aggregation behavior of nanoparticles in fluidized beds. Powder Technol 160(3):149-160

Hasselman DPH (1962) On the porosity dependence of the elastic moduli of polycrystalline refractory materials. J Am Ceram Soc 45(9):452-453. doi:10.1111/j.1151-2916.1962. tb11191.x

Hertz H (1881) Ueber die berhrung fester elastischer krper. J Reine Angew Math 92:156-171

Hu D, Zhuang J, Ding M (2012) A review of studies on the granular agglomeration mechanisms and anti-agglomeration methods. Key Eng Mater 501:515-519

Hutter J, Bechhoefer J (1993) Calibration of atomic-force microscope tips. Rev Sci Instrum 64(7):1868-1873. doi:10. 1063/1.1143970

Iwadate Y, Horio M (1998) Prediction of agglomerate sizes in bubbling fluidized beds of group c powders. Powder Technol 100:223-236

Johnson KL, Kendall K, Roberts AD (1971) Surface energy and the contact of elastic solids. Proc R Soc Lond Ser A 324:301-313

JPK (2009) Determining the elastic modulus of biological samples using atomic force microscopy. JPK Instrum. www.jpk.com

Jung J, Gidaspow D (2002) Fluidization of nano-size particles. J Nanopart Res 4:483-497
Kendall K, Alford NM, Birchall JD (1987) Elasticity of particle assemblies as a measure of the surface energy of solids. Proc R Soc Lond A 412(1843):269-283. doi:10.1098/rspa. 1987.0089

Kendall K, Weihs TP (1992) Adhesion of nanoparticles within spray dried agglomerates. J Phys D 25(1A):A3. http:// stacks.iop.org/0022-3727/25/i=1A/a =002

Khadilkar A, Rozelle PL, Pisupati SV (2014) Models of agglomerate growth in fluidized bed reactors: critical review, status and applications. Powder Technol 264(0): 216-228. doi:10.1016/j.powtec.2014.04.063, URL:http:// www.sciencedirect.com/science/article/pii/ S0032591014003817

Kovacik J (2001) Correlation between shear modulus and porosity in porous materials. J Mater Sci Lett 20(21):1953-1955. doi:10.1023/A:1013186702962

Kruis FE, Fissan H, Peled A (1998) Synthesis of nanoparticles in the gas phase for electronic, optical and magnetic applicationsa review. J Aerosol Sci 29(56):511-535. doi:10.1016/ S0021-8502(97)10032-5, URL:http://www.sciencedirect. com/science/article/pii/S0021850297100325

Kupkova M (1993) Porosity dependence of material elastic moduli. J Mater Sci 28(19):5265-5268. doi:10.1007/ BF00570076

Landolsi F, Ghorbel FH, Dabney JB (2013) Adhesion and friction coupling in atomic force microscope-based nanopushing. J Dyn Syst Meas Control-Trans ASME 135(1):011002

Laube J, Salameh S, Kappl M, Madler L, Ciacchi LC (2015) Contact forces between tio2 nanoparticles governed by an interplay of adsorbed water layers and roughness. Langmuir 31(41):11288-11295. doi:10.1021/acs.langmuir.5b02989

Liang Y, Ozawa M, Krueger A (2009) A general procedure to functionalize agglomerating nanoparticles demonstrated on nanodiamond. ACS Nano 3(8):2288-2296. doi:10. 1021/nn900339s

Li K, Chen Y (2014) Examination of nanoparticle-dna binding characteristics using single-molecule imaging atomic force microscopy. J Phys Chem C 118(25):13876-13882. doi:10. 1021/jp500737c

Limbach LK, Li YC, Grass RN, Brunner TJ, Hintermann MA, Muller M, Gunther D, Stark WJ (2005) Oxide nanoparticle uptake in human lung fibroblasts: effects of particle size, agglomeration, and diffusion at low concentrations. Environ Sci Technol 39(23):9370-9376

Lin DC, Dimitriadis EK, Horkay F (2007) Robust strategies for automated afm force curve analysisii: adhesion-influenced indentation of soft, elastic materials. J Biomech Eng 129:904-912

Mackenzie JK (1950) The elastic constants of a solid containing spherical holes. Proc Phys Soc Sect B 63(1):2-11. http:// stacks.iop.org/0370-1301/63/i=1/a=302

Madler L, Lall AA, Friedlander SK (2006) One-step aerosol synthesis of nanoparticle agglomerate films: simulation of film porosity and thickness. Nanotechnology 17(19):4783. http://stacks.iop.org/0957-4484/17/i=19/a=001

Martin RB, Haynes RR (1971) Confirmation of theoretical relation between stiffness and porosity in ceramics. J Am Ceram Soc 54(8):410-411. doi:10.1111/j.1151-2916.1971. tb12333.x 
Matsuda S, Hatano H, Muramoto T, Tsutsumi A (2004) Modeling for size reduction of agglomerates in nanoparticle fluidization. Aiche J 50(11):2763-2771

Maugis D (1992) Adhesion of spheres: The jkr-dmt transition using a dugdale model. J Colloid Interface Sci 150(1):243-269. doi:10.1016/0021-9797(92)90285-T, URL:http://www.scie ncedirect.com/science/article/pii/002197979290285T

Navrotsky A (2003) Energetics of nanoparticle oxides: interplay between surface energy and polymorphism. Geochem Trans 4:34-37

Norman LD (1971) Computer simulation of particulate systems. UNT Digital Library

Pabst W, Gregorova E, Ticha G (2006) Elasticity of porous ceramics - a critical study of modulus-porosity relations. J Eur Ceram Soc 26:1085-1097

Parveen F, Berruti F, Briens C, McMillan J (2013) Effect of fluidized bed particle properties and agglomerate shape on the stability of agglomerates in a fluidized bed. Powder Technol 237:46-52

Phani K, Niyogi S (1987) Elastic modulus-porosity relationship for si3n4. J Mater Sci Lett 6(5):511-515. doi:10.1007/ BF01739269

Picas L, Milhiet PE, Hernandez-Borrell J (2012) Atomic force microscopy: a versatile tool to probe the physical and chemical properties of supported membranes at the nanoscale. Chem Phys Lipids 165:845-860

Pimpang P, Zoolfakar AS, Wongratanaphisan D, Gardchareon A, Nguyen EP, Zhuiykov S, Choopun S, Kalantar-zadeh K (2013) Atomic force microscopy adhesion mapping: revealing assembly process in inorganic systems. J Phys Chem C 117(39):19984-19990. doi:10.1021/jp406210u

Quevedo JA, Omosebi A, Pfeffer R (2010) Fluidization enhancement of agglomerates of metal oxide nanopowders by microjets. AIChE J 56(6):1456-1468. doi:10.1002/aic. 12075

Quevedo JA, Pfeffer R (2010) In situ measurements of gas fluidized nanoagglomerates. Ind Eng Chem Res 49: 5263-5269

Radmacher M, Fritz M, Kacher C, Cleveland J, Hansma P (1996) Measuring the viscoelastic properties of human platelets with the atomic force microscope. Biophys J 70(1):556-567. doi:10.1016/S0006-3495(96)79602-9, URL:http://www.sci encedirect.com/science/article/pii/S0006349596796029

Rico F, Roca-Cusachs P, Gavara N, Farré R, Rotger M, Navajas D (2005) Probing mechanical properties of living cells by atomic force microscopy with blunted pyramidal cantilever tips. Phys Rev E 72:21-914. doi:10.1103/PhysRevE.72. 021914

Roduit CDGCSLFKSd, Sekatski S (2009) Stiffness tomography by atomic force microscopy. Biophys J 97:674-677

Rong W, Pelling AE, Ryan A, Gimzewski JK, Friedlander SK (2004) Complementary tem and afm force spectroscopy to characterize the nanomechanical properties of nanoparticle chain aggregates. Nano Lett 4(11):2287-2292. doi:10. 1021/n10487368

Salameh S, Schneider J, Laube J (2012) Adhesion mechanisms of the contact interface of tio2 nanoparticles in films and aggregates. Langmuir 28:11457-11464

Salameh S, Scholz R, Seo JW, Madler L (2014) Contact behavior of size fractionated $\mathrm{TiO}_{2}$ nanoparticle agglomerates and aggregates. Powder Technol 256:345-351
Schopf SO, Salameh S, Madler L (2013) Transfer of highly porous nanoparticle layers to various substrates through mechanical compression. Nanoscale 5:3764-3772. doi:10. 1039/C3NR34235B

Schwarz RB, Khachaturyan K, Weber ER (1997) Elastic moduli of gallium nitride. Appl Phys Lett 70(9):1122-1124. doi: $10.1063 / 1.118503$

Seville JPK, Willett CD, Knight PC (2000) Interparticle forces in fluidisation: a review. Powder Technol 113(3):261-268

Shabanian J, Jafari R, Chaouki J (2012) Fluidization of ultrafine powders. Int Rev Chem Eng 4:16-50

Sigdel K, Grayer JS, King GM (2013) Three-dimensional atomic force microscopy: interaction force vector by direct observation of tip trajectory. Nano Lett 13:5106-51111. doi:10.1021/nl403423p

Stiles RL, Willets KA, Sherry LJ, Roden JM, Van Duyne RP (2008) Investigating tip-nanoparticle interactions in spatially correlated total internal reflection plasmon spectroscopy and atomic force microscopy. J Phys Chem C 112:11696-11701

Tahmasebpoor M, de Martin L, Talebi M, Mostoufi N, van Ommen JR (2013) The role of the hydrogen bond in dense nanoparticle-gas suspensions. Phys Chem Chem Phys 15:5788-5793

Tanabe I, Tatsuma T (2012) Size- and shape-controlled electrochemical deposition of metal nanoparticles by tapping mode atomic force microscopy. J Phys Chem C 116(6):3995-3999. doi:10.1021/jp2123086

Tsai CJ, Pui DYH, Liu BYH (1991) Elastic flattening and particle adhesion. Aerosol Sci Technol 15(4):239-255. doi:10. 1080/02786829108959531

Vakarelski IU, Higashitani K (2006) Single-nanoparticle-terminated tips for scanning probe microscopy. Langmuir 22:2931-2934

van Ommen JR (2012) Manufacturing core-shell nanoparticles by atomic layer deposition in a fluidized bed. In: International symposium on chemical reaction engineering

van Ommen JR, Valverde JM, Pfeffer R (2012) Fluidization of nanopowders: a review. J Nanopart Res 14:737-766

Vinckier A, Semenza G (1998) Measuring elasticity of biological materials by atomic force microscopy. FEBS Lett 430(12):12-16. doi:10.1016/S0014-5793(98)00592-4, URL:http://www.sciencedirect.com/science/article/pii/S0 014579398005924

Wagh AS, Poeppel RB, Singh JP (1991) Open pore description of mechanical properties of ceramics. J Mater Sci 26:3862-3868

Wang JC (1984) Young's modulus of porous materials. J Mater Sci 19(3):809-814. doi:10.1007/BF00540452

Webber GB, Manica R, Edwards SA, Carnie SL, Stevens GW, Grieser F, Dagastine R, Chan DY (2008) Dynamic forces between a moving particle and a deformable drop. J Phys Chem C 112(2):567-574. doi:10.1021/jp076215d

Wyss HM, Franke T, Mele E, Weitz DA (2010) Capillary micromechanics: measuring the elasticity of microscopic soft objects. Soft Matter 6:4550-4555. doi:10.1039/C0 $03344 \mathrm{H}$

Xu D, Liechti KM, Ravi-Chandar K (2007) On the modified tabor parameter for the jkrdmt transition in the presence of a liquid meniscus. Colloid and Interface. Science 315:772-785 
Yan W, Li S, Zhang Y, Yao Q, Tse SD (2010) Effects of dipole moment and temperature on the interaction dynamics of titania nanoparticles during agglomeration. J Phys Chem C 114(24):10755-10760. doi:10.1021/jp102750k

Yao W, Guangsheng G, Fei W, Jun W (2002) Fluidization and agglomerate structures of $\mathrm{SiO}_{2}$ nanoparticles. Powder Technol 124:152-159

Yoshimura HN, Molisani AL, Narita NE, Cesar PF, Goldenstein $\mathrm{H}$ (2007) Porosity dependence of elastic constants in aluminum nitride ceramics. Mater Res 10:127-133
Zheng W, Ya-Pu Z (2004) Experimental investigation of the velocity effect on adhesion forces with an atomic force microscope. Chin Phys Lett 21:616-619

Zhou T, Li H (1999) Estimation of agglomerate size for cohesive particles during fluidization. Powder Technol 101:57-62 